\title{
La science citoyenne : Examen de son application comme outil de surveillance prodromique des maladies à transmission vectorielle
}

\author{
Hines $\mathrm{D}^{1^{*}}$, Sibbald $\mathrm{SL}^{1,2,3}$ \\ ${ }^{1}$ Schulich Interfaculty Program in Public Health, Université Western, London (Ontario) \\ ${ }^{2}$ Département de médecine, Université Western, London (Ontario) \\ ${ }^{3}$ School of Health Studies, Université Western, London (Ontario) \\ *Correspondance: dhines6@alumni.uwo.ca
}

\section{Résumé}

La science citoyenne désigne la collecte et l'analyse systématiques de données, la mise au point de technologies, l'analyse des phénomènes naturels et la diffusion de ces activités par des chercheurs sur une base essentiellement non professionnelle ou volontaire. Les applications mobiles fondées sur la science citoyenne offrent aux Canadiens un moyen de participer à la surveillance des maladies infectieuses.

Cet article présente des arguments en faveur d'une application mobile qui pourrait être utilisée pour améliorer la surveillance des maladies à transmission vectorielle au Canada. La maladie de Lyme est utilisée comme exemple en raison de son incidence croissante et du peu de renseignements en temps réel disponibles sur cette maladie. Les auteurs indiquent également comment cette application pourrait être conçue et utilisée de manière à inciter les utilisateurs finaux à la télécharger et à l'utiliser comme outil de santé publique. Si elles sont efficaces, les applications de ce genre pourraient servir de complément aux programmes de surveillance active, et aussi de moyen d'établir une communication bidirectionnelle entre les professionnels de la santé publique et les citoyens.

\section{Introduction}

Au cours de la dernière décennie, les changements climatiques et la santé publique sont devenus inextricablement liés(1). Selon un récent sondage, plus de $81 \%$ des Canadiens sont préoccupés par les risques pour la santé qui sont associés aux changements climatiques. Le même sondage a révélé que $49 \%$ des Canadiens estiment que les changements climatiques ont accru l'incidence des maladies infectieuses (2). Alors que le climat du Canada continue de se réchauffer, les populations de tiques et d'autres vecteurs commencent à augmenter, notamment dans des régions comme le sud de la Colombie-Britannique et de l'Ontario (3). La maladie de Lyme est la zoonose à transmission vectorielle la plus répandue en Amérique du Nord et elle est en hausse dans l'ensemble du Canada. En 2010, il a été estimé que $18 \%$ de la population de l'est et du centre du Canada vivait dans des régions où il y avait un risque de contracter la maladie de Lyme, et on s'attend à ce que ce pourcentage augmente $(4,5)$.

La tique aux pattes noires du genre Ixodes, connue pour être un vecteur de la bactérie Borrelia burgdorferi qui cause la maladie de Lyme, est présente dans des forêts caducifoliées ou mixtes partout au Canada (6). En plus de la maladie de Lyme, des cas d'autres maladies à transmission vectorielle rarement recensées au Canada commencent à apparaître plus près de la frontière canadienne. À titre d'exemple, en 2014, les Centers for Disease Control and Prevention (CDC) des États-Unis ont rapporté les premiers cas de deux maladies transmises par des tiques (le virus Powassan, connu pour causer des problèmes neurologiques à long terme, et l'anaplasmose, une maladie dont les symptômes typiques incluent la fièvre et des maux de tête) dans la région des Grands Lacs (7).

La surveillance des maladies infectieuses au Canada a permis de déterminer l'apparition et la propagation de maladies infectieuses telles que la grippe $A(H 1 N 1)$, la grippe $H 7 N 9$, le syndrome respiratoire aigu sévère (SRAS), la maladie de Lyme et d'autres maladies. L'augmentation des renseignements « en temps réel » disponibles sur ces maladies pourrait aider les responsables de la santé publique à mieux comprendre les risques d'éclosion et favoriser l'éducation du public. Idéalement, la collecte de renseignements en temps réel 
devrait s'inscrire dans un effort collectif. La récente éclosion de la maladie à virus Ebola a incité des chercheurs à examiner comment les activités des utilisateurs de téléphones intelligents pourraient être mises à contribution pour suivre la propagation de la maladie (8). À l'heure actuelle, toutefois, les activités de ces utilisateurs ne font l'objet que d'une surveillance rétroactive. La science citoyenne pourrait contribuer à documenter les processus et ainsi assurer une surveillance en temps réel de la propagation de la maladie (8).

La science citoyenne a été définie par Kinder comme « la collecte et l'analyse systématiques de données, la mise au point de technologies, l'analyse de phénomènes naturels et la diffusion de ces activités par des chercheurs sur une base essentiellement non professionnelle »(9) (traduction). Autrement dit, il s'agit d'observations faites par les citoyens ou de données recueillies sur base volontaire. Bien que l'expression « science citoyenne » soit relativement nouvelle, cette pratique n'a rien de nouveau $(10,11)$. Au cours des dernières années, des chercheurs ont utilisé la science citoyenne dans le cadre de leurs travaux pour corroborer des conclusions formulées dans des publications ou des présentations $(10,12,13)$.

L'intégration de la participation des citoyens à la surveillance de maladies à transmission vectorielle comme la maladie de Lyme, ou aux efforts de sensibilisation sur ces maladies, pourrait constituer une application nouvelle de la science citoyenne. Cela pourrait se faire par l'utilisation d'une application pour téléphones mobiles conçue de manière à recueillir des renseignements sur les habitats ou les aires de reproduction des vecteurs. Selon les auteurs, aucune application pour téléphones intelligents permettant la circulation bidirectionnelle de l'information sur les maladies à transmission vectorielle (de l'utilisateur final à l'administrateur, et vice versa) n'a encore été développée (14).

Ils proposent donc la mise au point d'applications fondées sur la science citoyenne pour mieux sensibiliser la population aux maladies à transmission vectorielle à déclaration obligatoire. De tels outils pourraient contribuer à mieux outiller les communautés et améliorer la protection de la santé publique. Cet article examine comment le pouvoir collectif des utilisateurs de téléphones intelligents pourrait être mis à profit pour favoriser la surveillance des maladies à transmission vectorielle et la sensibilisation de la population à ces maladies.

\section{Analyse}

Il est très difficile de suivre avec précision les maladies à transmission vectorielle, comme la maladie de Lyme, en temps réel (c.-à-d. période réelle durant laquelle un événement survient). Le suivi et l'identification des régions où les tiques à pattes noires Ixodes porteuses de $B$. burgdorferi sont endémiques exigent beaucoup d'heures et posent souvent problème, en raison des nombreuses autres demandes auxquelles doivent répondre les responsables de la santé publique. Ces problèmes pourraient toutefois être réduits en sollicitant l'aide des citoyens pour recueillir l'information requise. La mobilisation des citoyens, au moyen d'applications mobiles qui leur permettent d'observer des phénomènes naturels et d'être reliés à des recherches scientifiques sur une maladie à transmission vectorielle donnée, offre au public la possibilité de participer aux efforts de protection de la santé publique.

L'utilisation des téléphones intelligents est en hausse chez les Canadiens adultes (âgés de 18 à 65 ans). En 2013, un rapport de Google a estimé que $56 \%$ des adultes utilisaient un téléphone intelligent, comparativement à un pourcentage de $33 \%$ au début de l'année 2012 (15). Selon Google Canada, les utilisateurs de téléphones intelligents « utilisent leurs appareils mobiles pour changer la façon dont ils communiquent avec les autres et plus précisément pour tenter de comprendre le monde qui les entoure » (16) (traduction). Grâce à un usage accru des appareils mobiles et de leurs applications, les Canadiens sont mieux en mesure de contribuer à la protection de la santé de leurs communautés. Les applications mobiles fondées sur les concepts de la science citoyenne (9) présentent une option viable pour mobiliser les citoyens aux efforts de surveillance des maladies à transmission vectorielle et de sensibilisation à ces maladies.

\section{La science citoyenne, les applications mobiles et la santé}

La technologie des téléphones intelligents représente une prolongation naturelle de la science citoyenne, et offre en plus l'avantage de fournir des données en temps réel. Tout comme on a fait appel aux citoyens pour la numérisation de millions d'images à la recherche d'indices susceptibles d'aider à résoudre le cas de la disparition de l'avion de la compagnie Malaysia Airlines (17), l'intégration de la science citoyenne à une application mobile offrirait aux citoyens l'occasion de participer à la surveillance prodromique en temps réel (information sur la santé 
préalable au diagnostic) d'une maladie à transmission vectorielle et pourrait peut-être aussi servir à formuler des mises en garde concernant d'imminents problèmes de santé publique.

Un exemple d'une application conçue pour la surveillance des infections est l'application Flu near You (pas disponible en français) qui a été développée par HealthMap au Children Hospital de Boston (18). II s'agit d'un site communautaire de surveillance de la grippe, qui fournit au public des renseignements en temps réel. Un autre exemple est l'application de santé publique $I m m u n i z e O N_{1}$ qui a été conçue pour surveiller et suivre l'immunisation des Ontariens et leur fournir un outil pour les aider à se rappeler leur calendrier d'immunisation (19). Cette application met à jour les dossiers de vaccination, renseigne les utilisateurs finaux sur leurs calendriers d'immunisation et les informe des dangers potentiels liés à des vaccins défectueux ou à d'autres événements importants sur le plan de la santé publique grâce à l'utilisation de bannières. ImmunizeON est une excellente application de surveillance et de suivi, qui n'encourage toutefois pas la collecte et la transmission d'observations et de données. De plus, la fonction de communication entre les utilisateurs finaux et les responsables de la santé publique demeure limitée à l'envoi de courriels par l'entremise d'un site externe, et il n'y a aucune interface utilisateur permettant une communication directe au moyen de l'application. Une application fondée sur la science citoyenne élargirait la fonctionnalité de la communication, grâce à l'intégration d'une fonction permettant aux utilisateurs finaux de transmettre de l'information à la santé publique.

La fonction première d'une application sur la maladie de Lyme fondée sur la science citoyenne serait de communiquer aux citoyens des renseignements sur la manière d'identifier les tiques et leur habitat. L'application mobile sur la maladie de Lyme, qui a été conçue par le Bureau de santé de l'est de l'Ontario, fournit de l'information sur les symptômes de la maladie de Lyme, les caractéristiques physiques des tiques et les mesures à prendre en cas de morsure de tique (20), mais elle ne comprend pas de boucle de rétroaction pour aider à faire un suivi des endroits où les tiques sont présentes. Grâce à l'ajout d'une fonction de géomarquage (c'est-à-dire, la capacité de suivre l'emplacement géographique précis des tiques), l'utilisateur final pourrait étiqueter et prendre des photos de tiques susceptibles d'être porteuses du pathogène. La dernière couche de fonctionnalité consisterait à demander aux responsables de la santé publique de donner suite aux étiquettes ou photos en temps réel. Lorsque les responsables de la santé publique recevraient des envois, une équipe de professionnels qualifiés pourrait examiner l'information afin de déterminer s'il y a lieu de mener une enquête plus poussée ( $p$. ex. s'agit-il véritablement d'une espèce de tique porteuse du pathogène?). Pour autant que les auteurs le sachent, les applications actuellement disponibles n'ont pas de fonction grâce à laquelle les citoyens peuvent soumettre des photos ou des données.

La science citoyenne permet aux utilisateurs finaux d'interagir avec des professionnels de la santé et de soumettre des observations que les professionnels eux-mêmes ne pourraient obtenir. Dans l'application ImmunizeON, la surveillance prodromique des effets indésirables potentiels liés à des vaccins défectueux serait possible, grâce à l'intégration d'une fonctionnalité de communication bidirectionnelle (c.-à-d. Messagerie intégrée). Un certain nombre de projets de science citoyenne ont été lancés avec succès et ces projets illustrent bien les possibilités de ces applications; mentionnons notamment le projet National Moth Week 2014, qui encourage l'envoi de photos et de données pour alimenter des bases de données en ligne sur les papillons nocturnes, ainsi que le projet sur le desmocère à manteau lancé par Dan Duran, Ph. D., de la Drexel University (13).

En théorie, une application de surveillance fondée sur la science citoyenne, assortie de critères de diagnostic pour le vecteur en question, permettrait aux citoyens d'identifier en toute confiance les zones susceptibles d'abriter des tiques. Cette surveillance pourrait s'exercer lorsque des citoyens participent à des activités telles que la randonnée, la chasse, le jardinage, etc. dans des champs, des parcs ou des forêts situés à l'intérieur, ou à proximité, de régions à risque connues.

\section{Développement de l'application}

Selon le Mobile Movement Report de Google, $82 \%$ des utilisateurs de téléphones intelligents au Canada utilisent leur appareil pour faire des recherches et lire les nouvelles, et $27 \%$ s'en servent pour trouver des renseignements liés à la santé. Ces utilisateurs qui se servent de leur appareil pour trouver de l'information sur la santé constitueraient un auditoire cible idéal pour une application fondée sur la science citoyenne. La promotion de cette application devrait toutefois être faite auprès de toutes les personnes intéressées, car la science 
citoyenne ne veut pas uniquement un moyen d'atteindre des personnes ou des groupes qui ont des connaissances du domaine. Toute application fondée sur la science citoyenne doit être conviviale et doit pouvoir être utilisée par les utilisateurs même les plus novices.

\section{Facteurs à considérer}

Il y a plusieurs éléments clés à prendre en compte pour l'élaboration et le lancement d'une initiative de science citoyenne (21). Le premier concerne les mesures incitatives visant à encourager l'utilisation de l'application. La science citoyenne à des fins de sensibilisation et de collecte de données ou d'information est considérée comme une activité non professionnelle, distincte du travail rémunéré (9). La plupart des logiciels ou « gratuiciels » sont distribués sans frais pour les consommateurs, et comportent peu ou pas de restrictions d'utilisation (12). L'ajout de mesures incitatives (pécuniaires ou autres) à une activité de science citoyenne pourrait accroître la participation, mais pourrait également avoir des conséquences non voulues en favorisant par exemple une participation axée sur les gains financiers (22).

La « ludification », ou l'intégration d'éléments ludiques dans un contexte qui n'est pas lié au jeu (23), est un deuxième facteur. En rendant l'application amusante à utiliser, la ludification peut aider à accroître l'intérêt des participants et à maintenir leur engagement (24), ainsi qu'à attirer et à fidéliser de nouveaux utilisateurs (23).

Un troisième facteur est la promotion de l'application. La publicité dans les multimédias classiques (télévision, médias sociaux, publicités imprimées, etc.) et le Web 2.0 (comme les blogues et les forums Internet) sont des méthodes fiables de promotion. Les outils novateurs de marketing social, comme le fil RSS (fil de syndication ou de nouvelles - information de radiodiffusion vers des sites Web externes) sont également utiles pour attirer un large auditoire.

Il y a également d'autres questions à prendre en considération au moment d'élaborer une plateforme pour tout outil fondé sur la science citoyenne. Par exemple : Qui sera responsable de la gestion de l'information (un fournisseur ou une agence de santé publique)? Qui financera l'initiative (organisme local, national, privé)? Comment les résultats seront-ils diffusés à l'utilisateur final et au grand public?

\section{Conclusion}

On observe au Canada une augmentation des maladies à transmission vectorielle, qui est attribuable aux changements climatiques, à la modification de l'écologie et à d'autres facteurs. Une application mobile fondée sur la science citoyenne offrirait une tribune créative pour diffuser des outils d'apprentissage sur les vecteurs et les maladies à transmission vectorielle et pour interagir avec les Canadiens. La science citoyenne offre également la possibilité de responsabiliser les Canadiens et de maximiser leur capacité de contribuer à la création du savoir et à la surveillance de la santé. Si elle est efficace, l'application sur la maladie de Lyme pourrait servir de modèle pour le suivi d'autres maladies à transmission vectorielle comparables (p. ex. le virus Powassan et l'anaplasmose).

La science citoyenne gagne du terrain dans le domaine des applications mobiles et le milieu universitaire et elle offre également des possibilités dans le domaine de la santé publique. Selon les auteurs, aucune application de suivi et de surveillance des maladies à transmission vectorielle n'a encore été développée ou évaluée; nous ne pouvons donc pas garantir qu'une telle application serait efficace. Nous savons toutefois, grâce à l'étude de la science citoyenne et à l'utilisation d'autres applications comparables, que la participation de la communauté à des initiatives de ce genre offre de grandes possibilités en matière de surveillance et de sensibilisation.

Le présent article s'inscrit dans une discussion plus large sur la participation du public à la surveillance des maladies infectieuses. Durant une éclosion ou une pandémie, les citoyens peuvent se sentir impuissants et ils dépendent alors souvent des autorités sanitaires pour les guider et les protéger. L'utilisation accrue des appareils mobiles au sein de la population canadienne, combinée à la popularité croissante des applications mobiles de nouvelles et des médias sociaux, pourrait offrir un point d'accès pour accroître la capacité de surveillance des maladies infectieuses en temps réel. 


\section{Remerciements}

Nous remercions sincèrement Michel Deilgat pour son excellente contribution et son soutien à la rédaction des versions précédentes de cet article. Delaney Hines tient à remercier le programme de maîtrise en santé publique de la Schulich School of Medicine and Dentistry de l'Université Western, qui a organisé la possibilité de placement au Centre des maladies infectieuses d'origine alimentaire, environnementale et zoonotique de l'Agence de la santé publique du Canada, où la plupart des idées formulées dans le présent document ont été développées.

\section{Conflit d'intérêts}

Aucun

\section{Financement}

Aucun

\section{Références}

(1) Maibach EW, Nisbet M, Baldwin P, Akerlof K, Diao G. Reframing climate change as a public health issue: An exploratory study of public reactions. BMC Public Health. 2010;10(1): 299.

(2) Akerlof K, Debono R, Berry P, Leiserowitz A, Roser-Renouf C, Clarke K, Rogaeva A, Nisbet MC, Weathers MR, Maibach EW. Public perceptions of climate change as a human health risk: Surveys of the United States, Canada and Malta. Int J Environ Res Public Health. 2010;7(6):2559-606.

(3) Public Health Agency of Canada [Internet]. Lyme disease and other tickborne diseases: Information for healthcare professionals. Ottawa: Government of Canada; 2014. http://www.phac-aspc.gc.ca/id-mi/tickinfo-eng.php\#sec-2.2. Disponible en français : http://www.phac-aspc.gc.ca/id-mi/tickinfo-fr.php\#sec-2.2.

(4) Leighton PA, Koffi JK, Pelcat Y, Lindsay LR, Ogden NH. Predicting the speed of tick invasion: An empirical model of range expansion for the Lyme disease vector Ixodes scapularis in Canada. J Appl Ecol. 2012;49:457-464.

(5) Ogden NH, Radojevic M,Wu X, Duvvuri VR, Leighton PA, Wu J. Estimated effects of projected climate change on the basic reproductive number of the Lyme disease vector Ixodes scapularis. Environ Health Perspect. 2014;122(6): 63138. http://ehp.niehs.nih.gov/1307799/.

(6) Hatchette TF, Davis I, Johnston BL. Lyme disease: Clinical diagnosis and treatment. CCDR. 2014;40(11):194-208. http://www.phac-aspc.gc.ca/publicat/ccdr-rmtc/14vol40/dr-rm40-11/dr-rm40-11-lyme-1-eng.php. Disponible en français: http://www.phac-aspc.gc.ca/publicat/ccdr-rmtc/14vol40/dr-rm40-11/dr-rm40-11-lyme-1-fr.php

(7) Centers for Disease Control and Prevention [Internet]. Tickborne diseases of the U.S. Atlanta: Centers for Disease Control and Prevention, 14 July 2014. http://www.cdc.gov/ticks/diseases/.

(8) Talbot D. Cell-phone data could help predict Ebola's spread [Internet]. Boston: MIT Technology Review; 22 Aug. 2014. http://www.technologyreview.com/news/530296/cell-phone-data-might-help-predict-ebolas-spread/.

(9) Kinder J. Engaging Canadians in citizen science. Canadian Government Executive. 2014;20(4):15. http://www.canadiangovernmentexecutive.ca/category/item/1543-engaging-canadians-in-citizen-science.html.

(10) Iii F, Mims M. Amateur science: Strong tradition, bright future. Science. 1999;284(5411):55-56. http://www.sciencemag.org/content/284/5411/55.full.

(11) Feyerabend P. Science in a free society. 4th ed. Vol. 31. London: Verso; 1978. p. 385-90.

(12) Cornell Lab of Ornithology - Citizen Science Central [Internet]. Defining citizen science. Ithaca: National Science Foundation; 2014. http://www.birds.cornell.edu/citscitoolkit/about/definition.

(13) Scientific American [Internet]. Citizen science. New York: Scientific American; 2014. http://www.scientificamerican.com/citizen-science/.

(14) Benedict M, Geleta D. Development of ClickClinica: A novel smartphone application to generate real-time global disease surveillance and clinical practice data. BMC Medical Informatics and Decision Making. 2013;13(1):70-80. http://www.biomedcentral.com/1472-6947/13/70.

(15) Ipsos MediaCT [Internet]. Our mobile planet: Canada - Understanding the mobile consumer. Google, May 2013. http://think.withgoogle.com/mobileplanet/en/.

(16) Canadian Broadcasting Corporation [Internet]. Smartphone use way up in Canada, Google finds. 29 July 2013.

(17) Martinez M, Williams D, Simon D. Crowdsourcing volunteers comb satellite photos for Malaysia Airlines jet. Cable News Network, 12 Mar. 2014 
(18) American Public Health Association [Internet]. Flu near you: Do you have it in you? 20 Oct. 2014. https://flunearyou.org/about/.

(19) Wilson K, Atkinson K, Pluscauskas M, Bell C. A mobile-phone immunization record in Ontario: Uptake and opportunities for improving public health. J Telemed Telecare. 2014;1(5). http://www.ncbi.nlm.nih.gov/pubmed/25084770.

(20) Ontario Agency for Health Protection and Promotion (Public Health Ontario). Vector-borne diseases 2013 summary report. Toronto: Queen's Printer for Ontario; 2014. http://www.publichealthontario.ca/en/eRepository/Vector_Borne_Diseases_Summary_Report_2013.pdf.

(21) Archer G. Crowdsourcing: Improve healthcare with the brainpower of millions [Internet]. Action For Better Healthcare. 15 May 2014. http://actionforbetterhealthcare.com/crowdsourcing-improve-healthcare-brainpower-millions/.

(22) Kumagai D. Exploiting digital workers through global crowdsourcing. U of T News. Toronto: University of Toronto;11 Mar. 2014.

(23) Deterding S, Dixon D, Khaled R, Nacke L. From game design elements to gamefulness: Defining gamification, In: Proceedings of the 15th International Academic MindTrek Conference: Envisioning future media environments. 2011;9(15). http://gamification-research.org/2012/04/defining-gamification/.

(24) Von Bargen T, Zientz C, Haux R. Gamification for MHealth: A review of playful mobile healthcare. Stud Health Technol and Inform. 2014;225(28). http://www.ncbi.nlm.nih.gov/pubmed/25000057. 